\title{
CIENCIA, INCONMENSURABILIDAD Y REGLAS: CRÍTICA A THOMAS KUHN
}

\author{
Cristián Santibáñez Yáñez \\ Universidad Diego Portales \\ cristian.santibanez@udp.cl
}

\begin{abstract}
Resumen
Aún se utilizan insistentemente los conceptos de 'paradigma', 'inconmensurabilidad' y 'regla', tal como Thomas Kuhn lo propuso para referirse al modo en que se organizaría la ciencia. Lo problemático es que La estructura de las revoluciones científicas se cita como un caso de debate epistemológico, más que como un ensayo sobre la ciencia con perspectiva histórica. Se discute aquí el uso de Kuhn de estos conceptos para mostrar que esta posición no tiene otro apoyo más que una muy particular visión de la ciencia expuesta en el contexto de las décadas de 1950 y 1960 en Estados Unidos.
\end{abstract}

PALABRAS ClAVE: paradigma, inconmensurabilidad, traducción, seguir una regla, epistemología.

\section{Abstract}

The concepts of 'paradigm', 'incommensurability' and 'rule' are still widely used. Thomas Kuhn applied them to refer to the way in which science could be organized. The problem is that The Structure of Scientific Revolutions is quoted as a case of epistemological discussion, rather than a case of an historical perspective on science. In this paper Kuhn's use of these concepts is commented upon to show that this position has no other backing than a particular vision of science as exposed in the context of the 1950's and 1960's in the United States.

KeYwords: paradigm, incommensurability, translation, following a rule, epistemology. 


\section{Introducción}

$\overline{R A}$ La primera gran crítica a Thomas Kuhn versó sobre las múltiples y confusas definiciones que dio del término 'paradigma' en La estructura de las revoluciones científicas ${ }^{1}$. En la posdata de la edición de 1969, resumió en dos las posibles acepciones del concepto con el que quería describir la forma de trabajo de las ciencias, el desarrollo del conocimiento y, no menos importante en su empresa, el tipo social y psicológico del científico que se inserta en la práctica de un paradigma ${ }^{2}$.

Más allá del esfuerzo realizado por Kuhn por delimitar el sentido del concepto que reutiliza - pues como se comentará luego, proviene de la filosofía del siglo XVIII el primer uso sistemático del concepto 'paradigma'- lo que marca esta explicación en la posdata es un retroceso flagrante de Kuhn en sus intentos más rotundos por sentar un análisis del desarrollo del conocimiento, siendo el caso que, en muchas ocasiones, en este añadido se desdice de algunos alcances de su primera incursión, entra en contradicciones y agrega nuevos conceptos para abordar lo que quedó evidentemente desajustado en el comienzo.

Este trabajo, no obstante, trata discutir la idea general de basar el desarrollo de la ciencia en el modo, y supuesto, socio-psicológico de la práctica científica, es decir, se discute el exceso de la especulación aparentemente sociológica; y, más importante aún, se discute tanto el concepto de 'inconmensurabilidad' como el de 'regla' usado por Kuhn para analizar la estructura de la ciencia. Se sostiene aquí que, por un lado, la aplicación de la noción de 'inconmensurabilidad' no está sujeta a criterios epistemológicos relevantes y, por otro, que el desarrollo en torno al término de 'regla' en Kuhn funcionó sobre la base de una subdiferenciación, esto es, sobre la base de distinciones que no abarcaron todo lo implicado ${ }^{3}$.

1 Stephen Toulmin (1972) reporta que inmediatamente después de aparecer el libro de Kuhn en 1962, se produjo una ola de críticas en las que se desmenuzó su obra, lo que lo llevó a una primera defensa en 1965 en las presentaciones que diera en el Bedford College. En estas lecturas, de acuerdo con Toulmin, Kuhn debió retroceder en su inicial distinción entre ciencia normal y revolución científica, porque no encontraba un ejemplo genuino de una 'revolución científica'. Véase Toulmin (1972, pp. 96-130).

2 Para las dos acepciones, véase Kuhn (1996, p. 269).

3 Se debe señalar que el ejercicio crítico de este trabajo también es consecuencia de una constatación: la cita sostenida de este texto en tesis de pre y posgrado entre estudiantes y académicos, siendo el caso que, como se intentará mostrar, es un texto que entrega una imagen demasiado parcial de la actividad científica, cuyo concepto principal no explica rasgos epistemológicos medulares de la ciencia, y siendo también el caso que se puede refutar con el mismo tipo de especulaciones y generalizaciones. 


\section{Individuos, ambientes científicos y sentido común}

De acuerdo con Lenk ${ }^{4}$, es unánime entre los epistemólogos el aporte de Kuhn desde el momento en que éste enfatizó la inclusión de elementos históricos en la descripción del desarrollo científico. El paso de una teoría a otra es una secuencia histórica que se convierte en un problema epistemológico, en el sentido de que aparece la necesidad de observar las condiciones de desarrollo de las explicaciones y planificaciones temporales de la ciencia ${ }^{5}$.

Por otra parte, para algunos es un acierto la idea de 'inconmensurabilidad' para analizar cierta imposibilidad de traducción entre teorías. Con este concepto, Kuhn atacó al ambiente positivista reinante en su época. Para otros, como Davidson, por ejemplo ${ }^{6}$, es una imposibilidad epistemológica hablar de intraducibilidad o inconmensurabilidad entre teorías o lenguajes, pues ya el primer paso del intérprete por señalar que existe una tal inconmensurabilidad requiere que aquel conozca y asuma mucho acerca de las creencias de los involucrados, es decir, utilice una traducción o entendimiento previo.

El texto de Kuhn es una muestra generosa de las preocupaciones en la filosofía e historia de la ciencia en los años de 1950 y 1960, que estaba en plena discusión, por ejemplo, en torno a las nociones de verificacionismo y falsación. El hincapié de Kuhn en la inexistencia de un lenguaje neutro contribuyó al giro epistemológico que desbancó al Círculo de Viena.

Los problemas comienzan cuando se señala que es imposible la resolución de competencia entre paradigmas mediante pruebas, sino que ésta se debe a comportamientos sociales de elección de los involucrados, a un movimiento de persuasión, fuera de la discusión de la validez - normativa- de las teorías y del papel del contraste empírico, a pesar de que las propuestas dentro del propio giro epistemológico de los años de 1960 consideraban la discusión sobre la validez, como el racionalismo crítico o el pragmatismo metodológico.

Son varias las notas de Kuhn en este sentido. Llama sobre todo la atención, para comenzar, un rasgo de su posición que alude a la realización de un 'acto de terror' para resolver una disputa entre paradigmas: "En primer lugar, un paradigma no gobierna un tema de estudio, sino, antes bien, un grupo de practicantes. Todo estudio de una investigación dirigida a los paradigmas o a destruir paradigmas debe comenzar por localizar al grupo o los grupos de responsables" (276), dando a entender que ésta

4 Para un cuadro general de los debates epistemológicos de las décadas de 1960 y 1970, véase Lenk (1988, pp. 9-38).

5 A este respecto, valga señalar el esfuerzo contemporáneo de Steve Fuller por establecer una epistemología social (Social Epistemology originalmente en inglés) que dé cuenta de los aspectos de la producción y reproducción social de la ciencia. Véase Fuller (1988, 2003, 2006) y Fuller y Cullier (2004).

6 Véase el artículo de Davidson (2001, pp. 189-203) “De la idea misma de un esquema conceptual”. 
sería una forma para decidir la competencia entre paradigmas: la localización del oponente para destruir su paradigma, en vez de la discusión sobre los méritos teóricos y metodológicos de una teoría.

Si se sigue el primer uso académico del concepto, se puede señalar que un paradigma se refiere, precisamente, al estudio de estructuras que en sus funcionamientos gobiernan unidades que muestran recurrencia y patrón. Este fue el sentido que Georg Christoph Lichtenberg, en el siglo XVIII, le dio al término cuando hablaba de 'paradeigmata' en su estudio de las pautas formales del análisis gramatical en comparación con las pautas en el estudio teórico de la física.

Después de Lichtenberg, se le debe a las escuelas de lingüística el uso técnico extendido del término, el que fue importado desde las ciencias sociales. A pesar de que se le atribuye a Saussure la idea o definición contemporánea del término ${ }^{7}$, es el danés Louis Hjelmslev quien pone en la escena de las ciencias humanas este concepto en siglo XX. Junto a la escuela de Copenhague, las investigaciones de la escuela de Praga (Jakobson, Trubetzkoy, entre otros), el trabajo de la escuela soviética (la escuela de Kazán), e incluso los reportes de la escuela de Londres, son las que autorizan definitivamente el uso de la noción. En el marco de la glosemática de Hjelmslev (1974), 'paradigma' se refiere a un modelo de flexión nominal y verbal que rige las posibilidades de combinación en el eje de la selección, el sintagma.

Las reflexiones en filosofía del lenguaje de Wittgenstein, que formaron parte del panorama intelectual del contexto Kuhn, muestran un uso similar al de la lingüística del concepto de 'paradigma'. Wittgenstein, en el parágrafo 50 de las Investigaciones, expone la noción como un lugar donde ocurren comparaciones a partir de los juegos de lenguaje que se conciben como figuras de representación. Este lugar funciona a veces como un mecanismo de ostensión, en tanto recurso de ordenamiento. En los paradigmas, pareciera decir Wittgenstein, se relacionan distintos tipos de figuras asociadas a un cuadro de un juego de lenguaje. En el parágrafo 57, por ejemplo, muestra la imposibilidad de hacer desaparecer el color rojo antes de hacer desaparecer todo el paradigma de expresiones vinculadas, esto es, solo es posible erradicar un paradigma cuando su red de conexiones significativas desaparece, lo que en segundo término se relaciona con quienes emiten tales conexiones. En Sobre la certeza, Wittgenstein explica esto aludiendo a la forma en que se aprende un conjunto de proposiciones, sin mencionar, no obstante, el concepto de paradigma. En los parágrafos 141, 142 y 143, explícitamente señala que la creencia deviene en función de un sistema de proposiciones, y que los sistemas se mantienen unidos por sus premisas y no por quienes las utilizan.

7 La aparición del Curso de lingüistica general [1916] marcó la interpretación de muchos otros nuevos conceptos operativos en la lingüística posterior. Para una discusión general, véase Manoliu (1973, pp. 27-76). De acuerdo a Ducrot y Todorov (1983, p. 108) de Saussure habría hablado de paradigma en los cap. 5 y 6 de su Curso..., a través de la noción de 'grupos asociativos'. 
En un paradigma existe un conjunto de unidades que mantiene entre sí una relación virtual de vinculación, sustituibilidad o reemplazo; en la lingüística, en función del sistema de flexión; en la filosofía de Wittgenstein, en el cuadro de proposiciones; en semiótica, en el cuadro de signos. Los usuarios entran y salen del paradigma. No al revés. De allí que, si se quiere conservar la noción de paradigma, mejor es mencionar que es un cuadro de elementos que mantiene parecidos de familia entre sus componentes y que los gobierna, comanda, rige, en sus realizaciones. ¿Por qué un historiador de la ciencia, para hablar del desarrollo científico, no expresa el origen de su concepto central, las relaciones y diferencias con tal origen? ${ }^{8}$

Del mismo tenor especulativo es el siguiente pensamiento de Kuhn:

Como en las revoluciones políticas sucede en la elección de un paradigma: no hay ninguna norma más elevada que la aceptación de la comunidad pertinente. Para descubrir cómo se llevan a cabo las revoluciones científicas, tendremos, por consiguiente, que examinar no sólo el efecto de la naturaleza y la lógica, sino también las técnicas de argumentación persuasiva, efectivas dentro de los grupos muy especiales que constituyen la comunidad de científicos (152-3).

El año 1958, Toulmin publicó The uses of argument, donde examinaba y ponía en duda el carácter de la ciencia en tanto heredera directa del silogismo aristotélico, como dispositivo cuya lógica se asimilaba a la de las matemáticas. Más bien, Toulmin destacaba que la estructura de los argumentos de la ciencia se asemeja a la estructura de los argumentos en la jurisprudencia, donde juegan rol activo los mecanismos de atenuación y excepción de conclusiones. Antes que Kuhn, Toulmin había expuesto con mayor exactitud elementos de la estructura persuasiva de la empresa científica, pero no negó el hecho de que la ciencia se disputara a través de argumentos razonables, siendo la aceptación de la comunidad el resultado de las querellas presentadas vía argumentos o razones. La debilidad de los argumentos de la particular perspectiva de Kuhn para referirse al cambio de una constelación de creencias a otra entre los científicos de un campo, se observa hasta en el grado de inseguridad que proyecta y atribuye a la definición de tal cambio. Se destaca aquí con cursiva este hecho: "Para ser aceptada como paradigma, una teoría debe parecer mejor que sus competidoras; pero no necesita explicar y, en efecto, nunca lo hace, todos los hechos que se pueden confrontar con ella" (44). Aun cuando se hable en términos de "parecer", que ya es del todo equívoco, incluso el grado de incertidumbre asociado a la gramática del verbo requiere estándares razonables de decisión, esto es, persisten en torno al "parecer" mecanismos de presentación de pruebas y de sentido, que cubran con cierta razonabilidad una teoría, mostrando que una teoría tiene mayor consistencia que otra, que resiste mejor los embates de contrastación empírica que otra, que su normatividad obedece a

8 Aquí vale recordar que Kuhn estudió Física en Harvard, doctorándose en esta área en 1949. Posteriormente se dirigió a la Universidad de California, Berkeley, para hacer un posdoctorado en Historia de las Ciencias, que obtuvo en 1956. En 1964 se trasladó a la Universidad de Princeton; allí terminó su vida académica dictando clases sobre Historia de la Ciencia. 
rasgos contextuales específicos o variantes universales, que se pueden reconstruir y debatir.

En la misma dirección socio-psicológica se lee la insistencia de Kuhn:

... Las conversiones se producirán poco a poco hasta cuando, después de que los últimos en oponer resistencia mueran, toda la profesión se encuentre nuevamente practicando de acuerdo con un solo paradigma, aunque diferente. Debemos por consiguiente, inquirir cómo se induce a la conversión y cómo se encuentra resistencia... Los científicos individuales aceptan un nuevo paradigma por toda clase de razones y, habitualmente, por varias al mismo tiempo. Algunas de esas razones -por ejemplo, el culto al Sol que contribuyó a que Kepler se convirtiera en partidario de Copérnico- se encuentran enteramente fuera de la esfera de la ciencia. Otras deben depender de idiosincrasias de autobiografía y personalidad. Incluso la nacionalidad o la reputación anterior del innovador y de sus maestros pueden a veces desempeñar un papel importante (236-7).

Es probable que Kuhn no conociera los avances teóricos y conceptuales específicos de los análisis en retórica y teoría de la argumentación que en su contexto se estaban dando, no tan solo vinculados a Toulmin (en Inglaterra), sino también, por ejemplo, a Chäim Perelman y Olbrechts-Tyteca (en Bélgica) ${ }^{9}$. Se puede achacar al hecho de que las noticias académicas, en aquel entonces, cruzaban más lento el Atlántico, de lo cual, sin embargo, no hay antecedentes ciertos. Pero no es fidedigno que en su contexto inmediato, la academia americana de la década de 1950, no existiera un intento por analizar retóricamente a filósofos o científicos sociales. Por ejemplo, Kenneth Burke (1962), en el área de la literatura y la retórica, había hecho esfuerzos aplaudidos por sus colegas, pues mostraba claramente ciertos mecanismos retóricos en la posición, entre otros, de Bentham, Diderot, Pascal o Veblen.

Sin embargo, más llama la atención el hecho de que Kuhn reconociera que los científicos aceptan razones sesgadas por conveniencia nacionalista o por autoridad. ¿Cuál era el entorno inmediato de trabajo de Kuhn? Dicho de otra forma: es efectivo que en la empresa de convicción se usen todo tipo de argumentos, como los argumentos por autoridad ${ }^{10}$, pero también es cierto que estos mecanismos se delatan y se reconocen, ya desde Aristóteles, como movimientos falaces, y la propia competencia argumentativa del hablante-oyente permite denunciar esta estrategia.

En el capítulo La invisibilidad de las revoluciones científicas, Kuhn insiste en el papel que juega la autoridad para provocar cambios o hacer valer posiciones:

9 Véase Perelman y Olbrechts-Tyteca (2000) para las distinciones entre demostración y argumentación, entre tipos de argumentos, entre tipos de audiencias, ente otros tópicos vistos desde una perspectiva neo-retórica.

10 Precisamente, para un catastro de tipos de argumentos, véase Perelman y Olbrechts-Tyteca (2000, pp. 305-9), en particular, la distinción del argumento por autoridad basado en la estructura de la realidad. 
Como fuente de autoridad, acuden a mi imaginación, sobre todo, los libros de texto científicos junto con las divulgaciones y las obras filosóficas moldeadas sobre ellos. Estas tres categorías - hasta hace poco tiempo no se disponía de otras fuentes importantes de información sobre la ciencia, excepto la práctica de la investigación- tienen una cosa en común. Se dirigen a un cuerpo ya articulado de problemas, datos y teorías, con mayor frecuencia que al conjunto particular de paradigmas aceptado por la comunidad científica en el momento en que dichos libros fueron escritos... Para cumplir con su función, no necesitan proporcionar informes auténticos sobre el modo en que dichas bases fueron reconocidas por primera vez y más tarde adoptadas por la profesión. En el caso de los libros de texto, por lo menos, existen incluso razones poderosas por las que, en esos temas, deben ser sistemáticamente engañosos (213).

No queda en absoluto claro por qué el libro de texto, en tanto autoridad para el lector, tenga que ser sistemáticamente engañoso. La explicación de Kuhn de la forma en que funcionan los libros de textos, a los que agrega los textos de divulgación y las obras filosóficas, no arroja ningún análisis que el profano en las ciencias no adquiera por uso cotidiano. Supuestamente, y de acuerdo con la perspectiva socio-psicológica de Kuhn, el engaño del libro de texto se vería a través de la simplificación del lenguaje, la falta de citas a estudios genuinos y la persistente tendencia a normalizar el conocimiento, pues esto sería un requerimiento interno de la ciencia que empuja por mantener la tradición. Nunca ha habido claridad sobre esto. El mismo hecho de que Kuhn denunciara esto a través de su libro, muestra que existía ya en aquel entonces la posibilidad de encontrar publicaciones con distintos grados de erudición sobre temas varios que pusieran aviso sobre algún ilícito. No se sigue, ipso facto, que una vez que aparezca un libro de texto se esté frente a un engaño. En efecto, como otros tipos de publicaciones, los libros de textos son síntesis o manuales que intentan estar al alcance del lector, pero es tarea del editor, por ejemplo, filtrar los engaños posibles. Kuhn incluso agrega que "A menos que personalmente haya experimentado una revolución durante su propia vida, el sentido histórico del científico activo o el del lector profano de los libros de texto sólo se extenderá a los resultados más recientes de las revoluciones en el campo" (214). Olvida Kuhn los objetivos de un libro de texto, manual o síntesis: evidentemente, se economiza en el lenguaje, se simplifican ciertas explicaciones, se economiza el número de páginas y se busca que el lector tenga un texto que lo instruya de forma esquemática. Es parte de la práctica, incluso entre eruditos, consultar algo ameno, corto, pero no por ello equivocado, engañoso. El tamaño del libro de texto que contenga las variables históricas de la labor de un científico, sin mencionar el problema que supone ponerse de acuerdo en cuáles incluir, sería inmenso. Respecto de que si no experimenta una revolución en carne propia, el científico no tendría un cuadro adecuado de un cambio de constelación, es una arista psicológica que sobrepasa los límites de descripción del funcionamiento de un paradigma. Kuhn nunca señala qué entiende por historia y tradición, mal se puede juzgar si un libro de texto revela algo que nunca existió. ¿Cuál es el criterio? 
En el capítulo XII, La resolución de las revoluciones, se vuelve a la carga con este tipo de especulación ${ }^{11}$, acusando a los libros de texto de ser uno de los culpables del conservadurismo en la ciencia. También son los viejos profesores e investigadores, pues los que comienzan los cambios, a juicio de Kuhn, son las mentes jóvenes. Kuhn no muestra ningún dato que refuerce esta elucubración, se apela al supuesto sentido y práctica común de los científicos. Es verdad que existe una correlación mayor entre juventud y creatividad, pero no es privativa de la edad la producción del cambio. Wittgenstein, por ejemplo, que de joven apoyó el estado normal de la filosofía de su época, ya entrado en años cambió radicalmente su postura y con ello la constelación de creencias asociadas a sus temas.

Para Kuhn, las revoluciones científicas son invisibles. Cuesta pensar que una revolución sea invisible, es como el oxímoron 'movimiento paralizado': "Creo que hay excelentes razones por las que las revoluciones han resultado casi invisibles... -en parte, debido a razones funcionales importantes" (212). ¿Cuáles son esas razones funcionales importantes por las que se disimula una revolución? ¿Por dinero, por poder? Puede ser. Si fuera así, quedaría la triste imagen que la ciencia está gobernada por impulsos egoístas de la peor especie. Pero el hombre joven debe arreglárselas con la promesa y la fe, pues al respecto Kuhn sostiene que "El hombre que adopta un nuevo paradigma... deberá tener fe en que el nuevo paradigma tendrá éxito al enfrentarse a los muchos problemas que se presenten en su camino, sabiendo sólo que el paradigma antiguo ha fallado en algunos casos. Una decisión de esta índole sólo puede tomarse con base en la fe" (244). Se apela sin reservas a estados psicológicos particulares: un estado de expectativa inaugurado por el prometer y una conversión semi-religiosa posada sobre el acto de fe.

No es del todo claro desde qué experiencia social obtiene Kuhn sus generalizaciones. Por ejemplo, en el último capítulo, titulado Progreso a través de las revoluciones, se pueden discutir muchas de las observaciones vía comparaciones de contextos, épocas y grupos humanos. En este capítulo, Kuhn sostiene que es muy raro que comunidades científicas diferentes investiguen los mismos problemas. Pero puede ser que el caso fuera todo lo contrario: que lo común es que diferentes comunidades científicas investiguen los mismos problemas, no compartiendo puntos de vista. En lingüística sucede así; en semiología, la ciencia de los signos, también. La oscuridad de este tipo de generalización reposa en que no se sabe con exactitud qué es una comunidad científica para Kuhn: ¿investigadores de un mismo departamento académico?, ¿de una misma universidad o centro de investigación?, ¿de un país?

Al mismo tiempo, Kuhn reconoce que no dijo mucho sobre las ciencias sociales, pero cuando lo hizo equivocó el rumbo. Por ejemplo, sostuvo que los científicos sociales, a diferencia de los científicos naturalistas, eligen un problema en virtud de la importancia social de su solución ${ }^{12}$.

11 Véase pp. 224 y ss.

12 Véase pp. 253 y ss. 
Pero si se consultan los temas de tesis de pregrado o posgrado, de ayer y de hoy, de los departamentos de Sociología, Psicología, Antropología, Comunicación, Semiología, Etnología, Historia, etc., se verá que allí la variedad es lo que reina, que muy pocos de los investigadores sufren el apremio de escoger el tópico por una urgencia social. Incluso puede ser todo lo contrario, que el científico naturalista sienta el apremio por encontrar la nueva vacuna, el nuevo plan de evacuación frente a catástrofes, el diseño más óptimo de un puente, etc. Por otro lado, no es la academia la que mide el logro o el nivel de adecuación de un estudio, sino que son las instancias de poder administrativas del Estado, los gobiernos y las empresas, controladas por los presupuestos anuales, por las prioridades ideológicas y electorales, por la oportunidad del mercado, también en los años de 1950, las que permiten aplicar una teoría o certificar los resultados. Son tantos los científicos sociales que se preocupan por problemas no prácticos, que parece inaudito sostener que ellos siempre viajan con el peso de la noche social.

De acuerdo con Kuhn, "Cuando una comunidad científica repudia un paradigma anterior, renuncia, al mismo tiempo, como tema propio para el escrutinio profesional, a la mayoría de los libros y artículos en que se incluye dicho paradigma... Inevitablemente, estas observaciones sugerirán que el miembro de una comunidad científica madura es, como el personaje típico de 1984 de Orwell, la víctima de una historia reescrita por quienes están en el poder" (257). Tampoco es del todo claro que una comunidad repudie una 'constelación' anterior. Sin embargo, lo que puede estar equivocado con mayor seguridad, es que se renuncie a los libros del paradigma anterior, si se habla de educación, pues en las universidades e institutos se repasan los paradigmas en los cursos inferiores, y de allí el estudiante pasa a ser influenciado, si lo logra el profesor, por una perspectiva específica. Kuhn, al parecer, escribió su libro pensando la mayor parte del tiempo en 1984 .

Queda de manifiesto que las generalizaciones de Kuhn pueden ser contestadas con otras generalizaciones, pero ninguna de ellas se remite a críticas epistemológicas relevantes, del campo propio de la filosofía de la ciencia. Donde cabía hacer un ensayo de esta naturaleza, Kuhn no lo hizo. Por ejemplo, explayarse en el concepto de 'inconmensurabilidad'.

\section{Relativo a inconmensurabilidad}

Dado que para Kuhn un cambio de paradigma solo es posible con el inicio de una etapa de transición, porque la ciencia normal nunca provoca ni permite un cambio, el desarrollo científico no se da por acumulación, sino por transformación o reconstrucción de un campo. Solo la ciencia normal acumula, el cambio reconstruye. En esta reconstrucción existe un problema de entendimiento básico, pues se redefinen conceptos, se trastocan explicaciones y los científicos atrincherados en sus posiciones no intercambian una comunicación genuina, ni logran consenso. A esta relación de poco entendimiento, Kuhn la denomina 'inconmensurabilidad'. 
Frente a la acusación que se le hizo a Kuhn sobre su vago uso de la idea de inconmensurabilidad, la que fue entendida como elucidación conceptual de una imposibilidad total de comunicación entre quienes se sostienen en paradigmas distintos, el autor relativiza su posición en la posdata. En ella, Kuhn retrocede y postula que la distancia entre paradigmas solo se debe a vocabularios distintos, pero que los problemas no son meramente lingüísticos, sino también de valores, y lo que le resta a los investigadores es convertirse en traductores, dado que se reconocen entre sí como miembros de comunidades distintas.

Kuhn, en la posdata sigue apoyando esta posición con una explicación del papel del fenómeno de la traducción, enfatizando el hecho de que tal actividad, en todo caso, es una experiencia vicaria, que necesita como final del viaje el que los involucrados se conviertan en indígenas de la lengua (del paradigma en discusión), probablemente pensando en la experiencia antropológica-lingüística americana de mediados de la década de 1960 asociada a Gumperz y Hymes ${ }^{13}$.

En la nota de pie de página 17 en la posdata, al hablar de traducción, Kuhn interroga la posición de Quine, calificándola de incompleta, ya que este epistemólogo, según Kuhn, dice poco sobre la competencia del traductor para describir el mundo al que se aplica el lenguaje interpretado ${ }^{14}$.

Pareciera ser, no obstante, que lo medular de la posición de Kuhn, tomando incluso su postura de la posdata, es que los problemas de comunicación, o interrupción de la misma, no se solucionan con la mediación de una traducción, pues la conversión a una nueva matriz disciplinar ocurre vía persuasión, y ésta se realiza sin recurrir necesariamente a la traducción. Para Kuhn, el problema se soluciona cuando el candidato a la conversión pasa por un experiencia íntima -psicológica, nuevamente-y logra adquirir "la constelación de conjuntos mentales que por medio de la educación adquirirán los futuros miembros de la comunidad" (p. 311)

El nexo que hace Kuhn con Quine da la hebra para entender las especulaciones del físico. Quine describe el problema de la 'traducción', en vena estrictamente epistemológica y de teoría del lenguaje, con las nociones de 'traducción radical' y, luego, con la noción de indeterminación de la traducción ${ }^{16}$. Acorde con su perspectiva de rechazo a la distinción analítico-sintético, de distancia con los conceptos intensionales

13 Para una introducción apropiada a estos temas, véase Fishman (1995, pp. 33-40).

14 Puede ser un error de la edición que se consulta, pero en esta nota de pie de página, Kuhn cita el año de publicación de Word and Object de Quine como 1969, cuando la primera edición es de 1960, es decir, dos años antes de la publicación del propio texto de Kuhn, por lo que éste pudo conocer de antemano, y profundizar así, los argumentos de Quine, cuestión que no ocurre.

15 Llama poderosamente la atención una referencia que, se especula aquí en vena kuhniana, pudo haber sido el germen de la así llamada 'reprogramación neuro-lingüística': “Además, la traducción puede aportar puntos de entrada para la reprogramación neural, que por inescrutable que sea en este momento, debe hallarse subyacente en la conversión" (p. 311).

16 El ensayo Tres indeterminaciones de Quine, de 1988, que aparece en español en el texto compilatorio Acerca del conocimiento científico y otros dogmas (2001), ofrece una panorámica accesible. Para 
y de énfasis en un lenguaje extensional, con la primera noción Quine explica el problema de la traducción describiendo el trabajo del traductor, quien a partir de relaciones sencillas de referencias entre el entorno y las indicaciones del hablante, va construyendo una noción empírica del significado de lo implicado, para terminar con la competencia de emitir hipótesis respecto de otros significados y así crear un manual o inventario. Pero como es posible que dos traductores - dos polos en disputa, o dos paradigmas, o dos psicologías de dos paradigmas distintos- tengan sus manuales respectivos, y por lo tanto se haga difícil decidir cuál de ellos tiene el manual correcto, se hace necesario remitirse a las condiciones observables, y como las condiciones observables ya son categorizaciones, ocurre la inescrutabilidad de la referencia. La indeterminación de la traducción, entonces, es la imposibilidad de fijar referencia, una consecuencia de la opacidad referencial. Esta discusión Kuhn no la expone, aunque pareciera ser que él coincide con Quine, pero no menciona los aportes de éste.

Los problemas de esta posición, sin embargo, pueden ser discutidos con la perspectiva de Davidson $(2001)^{17}$, a través del argumento de la 'triangulación', que sostiene que en un proceso de comunicación o entendimiento lingüístico es necesario adoptar una línea imaginaria que una al hablante nativo, en este caso a los protagonistas de los paradigmas, con el objeto en disputa, y también otras dos direcciones que unan al traductor radical con el mismo objeto y su interlocutor. Esta estructura de entendimiento, que para Davidson es una consecuencia de la inseparabilidad de la teoría y el lenguaje, parte del supuesto de la maximización del acuerdo respecto de la verdad, esto es, que el traductor radical necesita partir de una teoría de la verdad para su propia lengua, asumida la verdad como resultado lógico (la convención V), y la interacción empírica producto de los asentimientos - de aceptación o rechazo- provocados en una comunicación. Davidson discute la posición de Kuhn en De la idea misma de un esquema conceptual, y a través de diferentes pasajes de este ensayo pone en tela de juicio el que podamos incluso hablar de mundos diferentes, sistemas conceptuales diferentes o inconmensurabilidad. Las tesis defendidas por Davidson son bastante categóricas. Davidson sostiene que la expresión de Kuhn de que los investigadores "trabajan en diferentes mundos" es una pluralidad metafórica, imaginada, puesto que hay cuando mucho un mundo, y compara la posición de Kuhn con la de Strawson, para quien uno puede imaginar mundos posibles, pero no reales, comparándolos con nuestros recursos descriptivos que se mantienen fijos. Sostiene Davidson: "Kuhn, por otra parte, nos dice que pensemos en diferentes observadores del mismo

estos comentarios, véase también la introducción de Rodríguez (pp. 9-32) a esta edición. Las explicaciones originales de Quine se encuentran en Word and Object de 1960.

17 Algunos de sus ensayos han sido reunidos en De la verdad y de la interpretación (2001) -edición en español-, en Subjective, Intersubjective, Objective (2002) -existe traducción al español, Cátedra (2003)-, y en Problems of Rationality (2004). Nótese que algunas de las ideas que Quine expresa en su texto de 1960 fueron discutidas, como el autor lo señala en notas de pie de página, con Donald Davidson. 
mundo que llegan a él con inconmensurables sistemas de conceptos. Los muchos mundos imaginados de Strawson se ven o se escuchan o se describen desde el mismo punto de vista; el mundo único de Kuhn se ve desde diferentes puntos de vista" (192). Hablar de puntos de vista diferentes, animados por mundo paradigmáticos disímiles, es un movimiento paradójico, porque para Davidson solo tiene sentido hablar de distintos puntos de vista si existe un sistema coordinado común en el que representarlos. La capacidad de observar la diferencia de esquemas conceptuales es solo posible si hay grados de intertraducibilidad entre los lenguajes implicado, ya que “... un hombre no puede perder la condición de hablar un lenguaje y retener al mismo tiempo el poder del pensamiento. Por lo tanto no es posible que alguien pueda ocupar un punto de observación para comparar esquemas conceptuales desprendiéndose temporariamente de los suyos propios" (191).

El cambio o retención de parte de un vocabulario - que en Kuhn se relacionaría con un paradigma que quiere ser abandonado- no es prueba suficiente de que se reemplaza u olvida un esquema conceptual, porque, según Davidson, la diferencia de vocabulario entre el viejo y el nuevo paradigma quizá solo se deba al pedestre y familiar hecho de que las oraciones son relativas al lenguaje al que pertenecen, y la diferencia entre los científicos de Kuhn, sostiene irónicamente Davidson, quizá se soluciona consultando un buen diccionario.

Los problemas de comunicación y traducción solo se pueden observar si se tiene a la mano una base común para la emisión e interpretación lingüística, y es la propia traducción, medianamente exitosa, la que permite ver tales fallos. Al respecto ensaya Davidson:

Un lenguaje puede contener predicados simples cuyas extensiones no son correspondidas por predicados simples, o incluso por ningún predicado, en algún otro idioma. Lo que nos permite lograr este objetivo en casos particulares es una ontología común a los dos lenguajes, con conceptos que individuan los mismos objetos. Podemos tener claros los fracasos de traducción cuando éstos son lo suficientemente locales, pues un marco de traducción generalmente exitosa proporciona lo necesario para hacer inteligibles los fallos (197).

Comprendemos al máximo las palabras y pensamientos de otros cuando interpretamos en una forma que optimice el acuerdo (esto incluye un margen, como dijimos antes, para el error explicable, como por ejemplo las diferencias de opinión). ¿Dónde deja esto un espacio para el caso del relativismo conceptual? La respuesta es, pienso yo, que mayormente debemos decir lo mismo de las diferencias de esquemas conceptuales que lo que decimos de las diferencias de creencias: incrementamos la claridad y el atractivo de las declaraciones de diferencia, sean de esquemas o de opinión, ampliando las bases del lenguaje compartido (traducible) o de la opinión compartida (202).

Queda medianamente de manifiesto que no es tan llano aceptar la idea de que entre paradigmas distintos no haya una base de entendimiento, sobre la base de un lenguaje básico común. 


\section{Relativo a reglas}

Particularmente en el capítulo V, titulado Prioridad de los paradigmas, Kuhn trata el tema de las 'reglas', sosteniendo una visión que desvincula la confección de paradigmas del seguimiento de reglas. Kuhn acepta el rol de éstas en la actividad de la ciencia normal cuando sus miembros buscan un tipo de tribunal que dirima sobre las anomalías que amenazan la estabilidad de la ciencia normal. Más allá de esto, las reglas en Kuhn no juegan ningún rol. También aborda este tópico en relación con el descubrimiento de reglas por parte de los historiadores de la ciencia. Sin embargo, como con las otras nociones analizadas en este trabajo, no hay una definición exacta, ni tampoco aproximada, del concepto de regla. Se acerca al punto de la siguiente forma:

La determinación de los paradigmas compartidos no es, sin embargo, la determinación de reglas compartidas. Esto exige una segunda etapa, de un tipo algo diferente. Al emprenderla, el historiador deberá comparar los paradigmas de la comunidad unos con otros y con sus informes corrientes de investigación. Al hacerlo así, su objetivo es descubrir qué elementos aislables, explícitos o implícitos, pueden haber abstraído los miembros de esa comunidad de sus paradigmas más globales, y empleado como reglas en sus investigaciones. Cualquiera que haya tratado de describir o analizar la evolución de una tradición científica dada, habrá buscado, necesariamente, principios y reglas aceptados de este tipo... Pero, si su experiencia tiene alguna similitud con la mía, habrá descubierto que la búsqueda de reglas es más difícil y menos satisfactoria que la de paradigmas $(80-1)$.

Vale comenzar por despachar las últimas líneas de la cita anterior. Probablemente, la dificultad en la búsqueda de reglas esté vinculada con la capacidad de los que investigan, la claridad en las prioridades, temas y proyectos, con la calidad de las hipótesis, del marco teórico previo, con el estado de avance de la disciplina en que se comienza una empresa como esta, etc. Sin embargo, no es nunca el argumento ad hominem el que logra mostrar las falencias de una argumentación. Más importante es, entonces, atender a la idea de que el historiador debe aislar los elementos que los miembros de una comunidad pudieran haber abstraído y empleado como reglas para sus investigaciones. Este punto es de suma importancia, pues se encuentra aquí una discusión clave en torno a las nociones de 'seguimiento de regla', por un lado, y 'observancia de reglas', por otro. Esta discusión ha sido abordada, en el marco de una disputa filosófica y lingüística, por la respuesta que dio Chomsky (1997, pp. 244-296) al argumento, o solución, de la paradoja escéptica de Wittgenstein, y que fue explicada por Kripke (1982, pp. 7-113), en función del acto de seguir una regla y la posibilidad de un lenguaje privado. Sobre esta discusión que clarifica el punto se volverá luego, en particular en torno a la idea del 'seguimiento de reglas'.

El objetivo que le confiere Kuhn al historiador de la ciencia, en relación con la identificación de reglas en el trabajo de los investigadores, a través de la sentencia "su objetivo es descubrir qué elementos aislables, explícitos o implícitos, pueden haber abstraído los miembros de esa comunidad de sus paradigmas más globales, y empleado 
como reglas en sus investigaciones", manifiesta una definición implícita sobre este concepto: pareciera ser que las reglas para Kuhn tienen el sentido de orientaciones o disposiciones generales carentes de normatividad. Por eso Kuhn insiste en lo innecesario de las reglas para la constitución de un paradigma, afirmando que :

En realidad, la existencia de un paradigma ni siquiera debe implicar la existencia de algún conjunto completo de reglas (82)... Los científicos trabajan a partir de modelos adquiridos por medio de la educación y la exposición subsiguiente a la literatura, con frecuencia sin conocer del todo o necesitar conocer qué características les han dado a esos modelos su status de paradigma de la comunidad. Por ello, no necesitan un conjunto completo de reglas. La coherencia mostrada por la tradición de la investigación de la que participan, puede no implicar siquiera la existencia de un cuerpo básico de reglas y suposiciones que pudieran descubrir una investigación filosófica o histórica adicional (84).

Lo que resulta difícil de asimilar en la explicación general de Kuhn es la relación que hay, finalmente, entre la noción de paradigma como un elemento que proporciona una solución concreta a un problema y que deviene como modelo y ejemplo, y la idea misma de regla. En términos generales, se espera de un modelo que represente, proyecte, mecanice, componga estructuralmente ciertos elementos de una entidad, objeto o hecho, del cual sea susceptible extraer regularidades que se puedan seguir y que a los ojos del usuario puedan convertirse en reglas para reconstruir ciertos estados iniciales de las entidades, objetos o hechos representados vía el modelo construido. Cuando cambian las circunstancias de los hechos u objetos, cambian los modelos. Así también, cuando se cambia la aplicación u omite una parte del modelo, se obtiene un error, una equivocación en la reconstrucción; en el mismo sentido, cuando no se sigue una regla, implícita o explícita, se obtiene un comportamiento inapropiado, que probablemente se entienda a medias, pero que puede explicarse a luz de del patrón que proyecta la regla. En la propuesta de Kuhn, el lector no sabe si la noción de regla está siendo pensada en un nivel de exposición teórica o de trabajo con metodologías. Las metodologías de investigación son modelos susceptibles de seguir, que contienen ciertas regularidades que conservan ciertos parecidos de familia. Las metodologías son reglas para el hábito científico. Los científicos saben que si no siguen, por ejemplo, ciertos procedimientos metodológicos, ciertas normas de publicación, ciertos estándares de participación académica, incluso ciertos hábitos regulados de comportamiento en las investigaciones, sus trabajos y artículos que defienden, profundizan y comunican constelaciones y ejemplares, corren el riesgo de no aparecer. La relación entre constelaciones y ejemplares, por un lado, y reglas, por otro, es más estrecha de lo que se piensa, pues a falta de un entendimiento conceptual - provocado por un supuesto problema de traducibilidad-, aun queda la revisión de la parte metodológica, del hábito o comportamiento en la investigación, para juzgar la calidad, fuerza o ámbito de aplicación de una propuesta o teoría.

Searle (1997, pp. 21-47) ha distinguido entre 'reglas constitutivas' y 'reglas regulativas', cuando expuso su punto de vista sobre la creación de los hechos institucionales a través del lenguaje. Esta distinción proporciona un marco adecuado de diferenciación conceptual del término 'regla' que en Kuhn faltó. Las 'reglas constitutivas' 
son aquellas que no solo regulan una actividad, sino que crean la posibilidad misma de existencia de ciertas actividades; por ejemplo, el ajedrez contiene reglas constitutivas, pues si no se siguen sus orientaciones, no se juega, realmente, ajedrez. Las reglas del ajedrez no son convenciones, en este sentido, pues no son arbitrarias. Caso distinto es, por ejemplo, la regla regulativa de manejar por la derecha de la calzada que regula la conducción, actividad de conducción que comenzó antes de la existencia de la regla en cuestión; en este caso, la regla tiene un perfil arbitrario, pues se dispuso por convención, por algún tipo de acuerdo para regular la actividad de condu$\operatorname{cir}^{18}$. Desde esta perspectiva, es posible que la actividad científica sea el caso de una actividad con reglas regulativas, ya que su deriva histórica así lo muestra, porque se trata de asumir ciertas orientaciones de comportamiento investigativo -profesionalpara el logro de resultados sistemáticos, puesto que el seguimiento de modelos para la solución de problemas con objeto de conocimiento ha existido con anterioridad. La ciencia en tanto actividad comporta un conjunto de orientaciones explícitas, desde que se inauguraron las universidades, más difusas en el pasado, pero más evidentes hoy.

Por otra parte, una discusión necesaria, que no desarrolla Kuhn del todo, es la relacionada con la existencia, búsqueda o invención de reglas en las ciencias naturales, por un lado, y las ciencias sociales, por otro; ligada a esta discusión se encuentra la problemática de la extrapolación de reglas y modelos de búsqueda de regularidades -e incluso vocabulario normativo-, desde las ciencias naturales -física y química, por ejemplo- a las ciencias del comportamiento humano -sociología, psicología, economía, antropología, etc. Searle discutió este tópico introductoriamente en Mentes, cerebros y ciencia (1994, pp. 81-96).

Si se sigue el desarrollo de Kuhn, el lector se encuentra con que el autor hace un vínculo no del todo claro entre racionalidad, paradigma y regla, pues menciona que los paradigmas, cuando son seguros, funcionan sin acuerdo sobre la racionalización. En efecto, a falta de un acuerdo se busca una autoridad, patrón, modelo o regla que suministre estabilidad que permita encontrar una solución. Muchas veces si tales autoridades, patrones, modelos o reglas consultadas no administran equilibrio para la solución de un problema, se cambian las reglas y se inaugura un camino alternativo. Pero ninguna nueva autoridad, patrón o regla tiene total independencia, es un hecho nuevo solo en función de su antecesor, herencia o fuente. Asimismo, es equivocado sostener que, en cualquiera de sus acepciones, los paradigmas cuando funcionan con seguridad no tienen acuerdo respecto de su racionalidad o racionalización. Es equivocado este juicio, porque racionalización en la ciencia, entre otras de sus posibles acepciones ${ }^{19}$, es un procedimiento general de administración de problemas y soluciones

18 Sobre la relación entre regla y convención, véase Searle, (1997, p. 66).

19 Para una discusión del concepto de racionalidad, racionalización y tipos de racionalidad, véase Lenk (1988, pp. 105-30). Para una discusión sobre la relación entre modernidad, racionalidad y racionabilidad, véase Toulmin (2003, pp. 294-309). 
con objeto de conocimiento, extensivo a todos los posibles paradigmas que contenga, que se construye sobre la base del registro de razones, evidencias, de la reducción de contradicciones formales y pragmáticas, esto es, es un proceso de auto-inclusión reflexiva de los sujetos y textos en la situación de argumentación y comunicación, procedimiento que funciona en términos de acuerdo implícito, como en la ciencia del siglo XIX, o explícito, tras el racionalismo crítico o el pragmatismo metodológico del siglo XX. La ciencia y los paradigmas que contiene, para la fortuna de todos, ya no es solo un tipo de comportamiento investigativo o académico, sino que contienen exigencias sujetas a orientaciones éticas y contextuales ordenadas por prioridades, es decir, racionalizada, o mejor, con racionabilidad, en el sentido de Toulmin (2003).

Luego Kuhn, todavía discutiendo sobre 'regla', critica a Wittgenstein: “A falta de un cuerpo pertinente de reglas, ¿qué es lo que liga al científico a una tradición particular de la ciencia normal? ¿Qué puede significar la frase inspección directa de paradigmas? El finado Ludwig Wittgenstein dio respuestas parciales a esas preguntas" (82).

Se conoce como la paradoja escéptica o la solución escéptica, el argumento de Wittgenstein respecto del seguimiento de reglas. De acuerdo a Kripke, el argumento se encuentra en los desarrollos de los parágrafos 137 al 242 de las Investigaciones, mientras que el argumento del lenguaje privado entre los parágrafos 243 al 315, y que en particular el 202 contiene el vínculo entre ambos, pues se sostiene allí que no es posible seguir privadamente una regla. Por cierto, no es que Wittgenstein no sostenga juicios sobre reglas en otros apartados, como en el 53 y 54 cuando relaciona juegos de lenguaje y reglas. Una cápsula del argumento en torno a 'seguir una regla' de Wittgenstein se puede obtener si leemos los apartados 199, 201 y 202.

Para Wittgenstein, según la interpretación más aceptada, en particular bajo la exégesis de Kripke, 'seguir una regla' es una costumbre que se puede rastrear en función de si las respuestas de una persona concuerdan con las propias, es decir, juzgar si un individuo está siguiendo una regla determinada en aplicaciones particulares es determinar si las respuestas de tal individuo coinciden con las mías en tal particular caso, lo que es lo mismo que decir que el individuo sigue, incluso comprende la regla, si logra funcionar con la forma de vida de la comunidad. Por esto, las reglas no son estados mentales, el individuo no tiene razones para seguirla, sino que se limita a hacerlo, es un salto al vacío, ciego. De este modo, y para la discusión en torno a la noción de regla y paradigma en Kuhn, es erróneo, desde la perspectiva de Wittgenstein, pedir a los miembros del paradigma que mantengan la coherencia mostrada por un sistema de investigación, paradigma, con exclusión de las reglas, pues los miembros, en tanto participes de una comunidad, solo se limitan a replicar en sus comportamientos, en sus usos, la reglas que mantienen la coherencia del grupo del que son parte.

El criterio de Wittgenstein para juzgar si alguien ha seguido una regla es la resolución correcta de, por ejemplo, un problema, es decir, el resultado de una operación. Esto es, un criterio externo. El proceso práctico de aplicación de una regla puede estar acompañado de distintos procesos mentales del individuo, de allí que no se pueda decir que el 'seguir una regla' esté especificado por un estado mental determinado. De este modo 'seguir una regla' es una aplicación competente. Un punto relacionado 
estrechamente con esta posición de Wittgenstein, es la distinción que se hace entre enunciar una oración en primera persona, del tipo "ya sé seguir la regla", que no cuenta como una descripción de un estado interno, y enunciar una oración en tercera persona, del tipo "ya sabe seguir..." dicha, por ejemplo, por un profesor, que cuenta como síntoma de un criterio externo de evaluación que remite a una forma de vida comunitaria a raíz del reconocimiento público.

Con estos elementos se puede aventurar una explicación aproximada de por qué el de Wittgenstein es un argumento que se denomina la solución o paradoja escéptica. Cuando intento explicar el uso de la regla a través de mi experiencia, no encuentro razones, su aplicación es ciega, pero cuando hablo de otro, la explicación tiene sentido, pues existen condiciones de justificación de la regla: lo comunitario. En este último sentido, además, la regla es una cuestión de normatividad, no de contingencias. Al preguntar cómo o por qué se sigue una regla, no se pregunta por hechos, sino por justificaciones.

Chomsky discute la posición global de Wittgenstein, y lo hace mostrando cómo habría que juzgar el seguimiento de regla en Robinson Crusoe que no pertenece a ninguna comunidad. El ejemplo también es tratado por Kripke (1982, p. 110). La reflexión y crítica de Chomsky se puede resumir como sigue.

En Chomsky, la posibilidad del seguimiento de reglas vía un uso privado tiene dos explicaciones. Una proveniente de una perspectiva científico-lingüística, y otra, desde un ángulo filosófico-ético. La primera viene a sostener que dado que poseemos una información genética para la producción de lenguaje, y ésta se manifiesta en distintas lenguas, y distintos hablantes pueden potencialmente hablar esas distintas lenguas, entonces la facultad se expresa de una forma mínima generalizada en los individuos de la especie para que estos estén en condiciones de expresar la infinidad de lenguas; a esta forma mínima se la agrupa en principios, susceptibles de ser observados en regularidades descriptivas; los principios, a su vez, como Muévase $\alpha$, se 'parametrizan' por la manifestación, es decir, por las lenguas particulares, por las formas de vida de la gramática; así, el conjunto de principios constituye la 'Gramática Universal', y la adquisición de una lengua particular consiste en el proceso de fijación de los parámetros a partir de la exposición a un conjunto reducido de datos en un medio lingüístico concreto y en un período de tiempo relativamente breve. El conjunto de principios de la 'gramática universal' con sus parámetros debidamente fijados es lo que constituye la 'gramática nuclear' (core grammar) de una lengua particular. Así, como constituyente de la especie biológica, la gramática nuclear pone a disposición una serie reglas que se pueden abstraer bajo un programa de investigación que conduzca a la explicación de la facultad del lenguaje y describa los mecanismos de producción de expresiones estructuradas recurrentes en un individuo, un grupo, una comunidad. En términos éticos, además, esta formulación científica recoge el hecho de la libertad humana de atribución de regla, reconocimiento del otro y aceptación de nuestra capacidad de distinción. Si se le atribuyen reglas a alguien, a su comportamiento, práctica o capacidad, se hace porque, entre otras cosas, se reconoce a ese individuo como característico de la especie, que exhibe los aspectos de la voluntad y potencial de elección que permiten, entre otras posibilidades, el manejo creativo del uso lingüístico 
y otros indicios de inteligencia, y, evidentemente, como sostiene Chomsky citando a Cordemoy, "porque te pareces a mi" 20 .

\section{Conclusiones}

Lo que ha quedado demostrado, o al menos esa ha sido la intención y el esfuerzo, es que los acercamientos de Kuhn a los conceptos de paradigma, inconmensurabilidad y regla han sido deficientes, empedernidamente especulativos y falto de distinciones clave. Por una parte, Kuhn no desarrolla un juicio histórico del concepto mismo de paradigma, a pesar de que su perspectiva es histórica; por otra, explica los conceptos de inconmensurabilidad y regla, debatidos profusamente en filosofía de la ciencia, sin considerar a cabalidad lo que está implicado en los debates entre los filósofos. Así, llama la atención que entre los académicos e investigadores de las ciencias sociales se le cite como una autoridad en la discusión epistemológica.

Es cierto que las investigaciones, en cualquier disciplina, no maduran solo por la obtención de datos, hechos o información nueva, sino que especialmente sobre la base de la interpretación de tales datos, el establecimiento de relaciones entre ellos, vía la exposición de preguntas e hipótesis arriesgadas -en realidad, los propios hechos nuevos aparecen por un ejercicio inicial a tientas; en esto juega un rol importante aventurar lecturas, proponer direcciones heterodoxas y especular. Dicho de otra forma, y como sostiene Derek Bickerton, "la especulación es un componente vital de la ciencia", sin olvidar además que "la especulación es un equipaje ligero, es fácil deshacerse de él y volver a intentarlo" 21 .

No obstante, la interpretación motivada solo por la especulación, esto es, por la improbabilidad, no significa una fe ciega en la aventura, ni menos pensar que el riesgo solo está en función de una posición personal. Una vez que el contexto de uso y justificación nos impele a una aclaración importante de nuestra perspectiva, incluso a la atenuación de la misma, la especulación cumple su rol retirándose. La posdata de Kuhn, en este sentido, es un acto falto de reconocimiento del equívoco inicial.

20 Ha quedado al margen toda la exposición que hace Chomsky sobre la 'observancia de reglas', la atribución de las mismas por parte del observador o científico, y el conocimiento de reglas; para esto último véase Chomsky (1997, pp. 266-96). Los desarrollos de Chomsky de estos últimos temas supera los objetivos de estas líneas por la variedad de tópicos implicados. 


\section{Referencias bibliográficas}

Burke, Kenneth (1962), A Rhetoric of Motives. Berkeley, Los Angeles, London: University of California Press.

Calvin, W. y D. Bickerton (2001), Lingua ex machina. La conciliación de las teorías de Darwin y Chomsky sobre el cerebro humano. Barcelona: Gedisa.

Chomsky, Noam (1997), El conocimiento del lenguaje. Barcelona: Altaya.

Davidson, Donald (2002), Subjective, Intersubjective, Objective. New York: Oxford UP.

(2004), Problems of Rationality. New York: Oxford UP.

(2001), De la verdad y de la interpretación. Fundamentales contribuciones a la filosofía del lenguaje. Barcelona: Gedisa.

Ducrot, O. and T. Todorov (1983), Encyclopedic Dictionary of the Sciences of Language. Baltimore and London: The Johns Hopkins UP.

Fishman, Joshua (1995), Sociología del lenguaje. Madrid: Cátedra.

Fuller, Steve (1988), Social Epistemology. Bloomington and Indiana: Indiana UP.

(2003), Kuhn vs. Popper. The Struggla for the Soul of Science. Cambridge: Icon Books. Ltd.

(2006), The New Sociological Imagination. New York: Sage Publications

Fuller, S. and J. Cullier (2004), Philosophy, Rhetoric and the End of Knowledge: A new Beginning for Science and Technology. Hillsdale, N.J.: Lawrence Erlbaum Associates.

Hjelmslev, Louis (1974), Prolegómenos a una teoría del lenguaje. Madrid: Gredos. Janik, A. y S. Toulmin (1998), La Viena de Wittgenstein. Madrid: Taurus.

Kripke, Saul (1982). Wittgenstein. On Rules and Private Language. Cambridge, Mass: Harvard UP.

Kuhn, Thomas (1996), La estructura de las revoluciones cientificas. Santiago: Fondo de Cultura Económica.

Lenk, Hans (1988), Entre la epistemología y la ciencia social. Barcelona: Alfa.

Manoliu, Marta (1973), El estructuralismo lingüístico. Madrid: Cátedra.

Perelman, Ch. and L. Olbrechts-Tyteca (2000), The new Rhetoric. A treatise on Argumentation. Notre Dame: University of Notre Dame Press.

Quine, W. (2001), Acerca del conocimiento científico y otros dogmas. Barcelona: Paidós.

(2001), Palabra y objeto. Barcelona: Herder.

Saussure, F. de (1945), Curso de lingüistica general. Buenos Aires: Losada.

Searle, John (1997), La construcción de la realidad social. Barcelona: Paidós.

Toulmin, Stephen (1958), The uses of argument. Cambridge: Cambridge UP. 
(1972), Human Understanding. The Collective Use and Evolution of Concepts. Princeton, NJ: Princeton UP. sula. (2001), Cosmópolis. El trasfondo de la modernidad. Barcelona: Penín(2003), Retorno a la razón. Barcelona: Península.

Wittgenstein, Ludwig (1997), Sobre la certeza. Barcelona: Gedisa. (2002), Investigaciones filosóficas. Barcelona: Crítica. 